\title{
Estressores Sociais da Hipertensão em Comunidades Carentes
}

\author{
Miguel Angel Gandarillas ${ }^{123}$ \\ Universidad SEK, España \\ Sheila Gonçalves Câmara \\ Universidade Luterana do Brasil, Canoas \\ Helena Scarparo \\ Pontifícia Universidade Católica do Rio Grande do Sul
}

\begin{abstract}
Resumo
A hipertensão arterial tem sido estudada sob vários enfoques. As pesquisas demonstram a conexão entre os determinantes sociais e as manifestações da doença. Este estudo trata dos estressores sociais de hipertensão na Vila Nossa Senhora de Fátima, Porto Alegre, Brasil. Relata e discute os resultados do trabalho realizado junto a esta população, no qual se examinam variáveis constitucionais, sociais e fisiológicas. O exame da literatura sobre o tema e a comparação com um grupo controle, advindo de estudo semelhante com população de outro contexto social, mostrou a influência dos estressores sociais no processo de desenvolvimento da hipertensão. As conclusões alcançadas permitem algumas recomendações de ação no sentido da construção da cidadania como instrumento de participação e promoção de saúde. Palavras-chave: Hipertensão; estressores sociais; classe social; qualidade de vida.
\end{abstract}

\section{Social Stressors of High Blood Pressure in Deprived Communities}

\begin{abstract}
The origins of systemic high blood pressure has been studied from a wide array of different approaches. Researches suggests a very close connection between social environment stressors and all the manifestations of the disease itself. The present study deals with relevant social stressors that might be related to the high rates of high blood pressure found among the inhabitants of Vila Nossa Senhora de Fátima, a poor neighborhood in Porto Alegre, Brasil. Results, when compared to those comming from samples from different contexts of the same city, suggest an important relationship between certain social circunstances and the evolution of the hypertension desease. The discussion points to the importance of building up ciitzenship as a tool for health participation and promotion.

Keywords: High blood pressure; social stressors; social status; quality of life.
\end{abstract}

A hipertensão arterial crônica (chamada também sistêmica ou essencial) é reconhecida como tal através de níveis excessivamente altos de pressão arterial, que se mantêm através do tempo. Este tipo de manifestação orgânica mostra uma incidência crescente, em âmbito mundial, nos últimos tempos, apresentando, inclusive, um incremento exacerbado em relação a outras enfermidades, de tal forma que já está sendo considerada a enfermidade de nosso século.

Apesar de intensas pesquisas durante as últimas décadas, ainda não se conhecem bem quais são os fatores sociais mais relevantes que farão com que uma pessoa desenvolva hipertensão e outra não. Talvez isto se deva à forma imperante de investigação, de tipo linear e unidimensional, que prioriza os fatores genéticos, constitucionais ou biológicos, em detrimento da globalidade da pessoa inserida em um contexto social específico. Embora existam muitas evidências acerca de fatores como herança genética, idade, obesidade, tabagismo e alcoolismo, estes falham em dar uma explicação suficientemente satisfatória para o fenômeno da hipertensão. Harrap (1994) indica várias concepções

\footnotetext{
${ }^{1}$ Endereço para correspondência: Universidad S. E. K. - Segóvia. C/ Cardenal Zúñiga, 12, 40003, Segovia, España. E-mail:mgandarillas@sekmail.com

2 Agradecimentos: Agradecemos ao Dr. Paolo Machado, médico da Unidade de UTI Coronária do Hospital São Lucas, pela sua colaboração nesta pesquisa, através da utilização dos dados de seu trabalho para o estudo comparativo.

${ }^{3}$ Apoio Institucional ao Projeto: O presente estudo é resultado do convênio de intercâmbio de pesquisadores e estagiários entre a Pontifícia Universidade Católica do Rio Grande do Sul (Brasil) e a Universidad Autónoma de Madrid(Espanha).
}

da investigação médica que induzem a preponderância dos fatores de herança, colocando em segundo plano as variáveis ambientais. Dentre estas concepções, está a crença de que as variáveis genéticas e ambientais influem, na mesma proporção, em todo qualquer tipo de grupo social.

Esse reducionismo, que tende a excluir características do ambiente social e econômico, pode estar subjacente à falta de um conhecimento profundo e global sobre como se desenvolve a hipertensão. A identificação de fatores sociais relevantes pode ajudar-nos a entender o que ainda não tem sido explicado a partir de um modelo puramente médico. Ainda, talvez variáveis consititucionais e biológicas estejam relacionadas com a hipertensão arterial, não apenas por seu efeito fisiológico, mas também por estarem associadas a características de tipo psicossocial.

$\mathrm{Na}$ literatura científica podem ser encontrados modelos teóricos explicativos sobre como os fatores sociais afetam o desenvolvimento da atividade psico-fisiológica (Cacioppo, 1994; Kemper, 1986). O sistema nervoso autônomo (responsável direto pela pressão sangüínea) é o encarregado de acomodar o corpo a cada mudança decorrente de circunstâncias ambientais e sociais. Dessa forma, sua atividade é, até certo ponto, um reflexo da ação sócio-cultural sobre as pessoas (Kemper, 1986). Por isso, também poder-se-ia esperar que normas e circunstâncias sociais conflitivas e estressantes refletissem-se no desenvolvimento de funcionamentos contraditórios dos diversos organismos do corpo. Esses acarretariam em disfunções fisiológicas, como é o caso da hipertensão crônica. 
As investigações empíricas têm demonstrado a importância de algumas variáveis ambientais sobre a ativação psicobiológica (cf. modelos propostos, p. ex., por Cacioppo, 1994; Gray, 1971, 1981). Particularmente, os modelos desses autores sugerem que uma diminuição no controle fisiológico, em função da redução da internalização de fontes de reforço (ou apoio social), faz com que o sistema parassimpático diminua sua ativação cronicamente e o simpático tenha maior possibilidade de expandir-se através de barreiras cognitivas. Nesse sentido, é bem conhecido o papel do sistema simpático na manutenção da hipertensão (ConsolimColombo \& Krieger, 2000). Esse fatores nos permitem hipotetizar que variáveis relacionadas a barreiras sociais podem estar diretamente associadas à hipertensão. De fato, fatores como o status sócioeconômico (Costa, 1983; Krantz, 2002), as experiências positivas vs. negativas na vida do indivíduo (Theorell \& Emlund, 1993), baixos níveis de controle social (Bosma \& cols. 1997; Karasek \& Theorell, 1990), ou baixos níveis de apoio social (Knox, 1993; Krantz, 2002) aparecem diretamente relacionados com a incidência da hipertensão. Cabe ressaltar, ainda, a importância dessas variáveis sociais na infância, como preditores diretos dos padrões emocionais psicofisiológicos que apresentará o adulto (Fowles, 1980; Kalimo \& Vuori, 1993; Knox, 1993; Larsen \& Ketelaar, 1991; Polanky, Gaudin, Amons \& Davis, 1985; Raine, 1988; Theorell \& Emlund, 1993; Whiting \& Whiting, 1975). De forma indireta, as variáveis sociais na infância influenciam no desenvolvimento de padrões de personalidade ligados ao estresse mental associado à hipertensão (De Oliveira, 2000).

Ainda que teoricamente se ressalte a importância dos fatores sociais no desenvolvimento de patologias fisiológicas, nos poucos estudos rigorosos, os autores, tipicamente, costumam concentrar-se em apenas uma ou duas características sociais que incidem no desenvolvimento da hipertensão crônica, como falta de apoio social e violência (Linden, Chambers, Maurice \& Lenz, 1993), nível econômico baixo (Kalino \& Vuori, 1993), cultura de pobreza (Anderson, McNeilly \& Myers, 1991), ou caracteristicas sóciofamiliares no período da infância (Erickson, Egeland \& Pianta, 1989; Peisner, 1989).

Estudos realizados por Dressler e Santos (2000), na realidade brasileira, refletem a importância da estrutura sócio-cultural concreta e o estilo de vida almejado culturalmente sobre a pressão arterial. Nesse sentido, os autores propõem um modelo, denominado consonância cultural, que tenta equilibrar a importância conferida à percepção do indivíduo em relação ao estresse e o contexto sócio-cultural onde este estresse se manifesta, enfatizando as formas de afrontamento desenvolvidas pela pessoa e os modelos culturais de vida que compartilha. O estilo de vida, na medida em que se aproxima do ideal almejado e compartilhado socialmente, torna-se um mediador fundamental entre as variáveis biológicas, individuais, contextuais e os níveis de saúde do indivíduo. Os datos revelam que quanto maior a consonância cultural que tem o indivíduo, menores os seus níveis de pressão sangüínea (Dressler, Balieiro \& Santos, 2001).

No caso da pressão arterial, o modelo de consonância cultural explica quase $10 \%$ das diferenças entre indivíduos, demonstrando que o estilo de vida, quanto mais próximo ao ideal cultural, melhor será enquanto mediador entre saúde e variáveis sócio-econômicas, tornando-se independente, inclusive, de status sócio-econômico, dieta e variáveis psicossociais (Dressler, Balieiro \& Santos, 1998).

Baseados nestes aspectos, realizamos uma pesquisa exploratória, com enfoque global e interdisciplinar, incluindo uma ampla gama de variáveis constitucionais e, especialmente, sociais, econômicas e culturais de uma comunidade que apresenta graves estressores sócioeconômicos, como é o caso da Vila Nossa Senhora de Fátima, em Porto Alegre, Brasil. A precária situação desta comunidade favelada propiciaria a identificação de estressores sociais relacionados à hipertensão, além de possibilitar uma melhor intervenção e prevenção desta doença entre seus moradores, bem como entre moradores de outras comunidades com características similares.

A pesquisa surgiu do trabalho junto ao Campus Aproximado da Pontifícia Universidade Católica do Rio Grande do Sul (PUCRS), que, como posto de saúde, presta atendimento aos moradores da Vila. No caso da hipertensão arterial, a proposta surgiu em seguida ao I Curso de Prevenção de Pressão Alta (realizado no Campus Aproximado da PUCRS), de forma a dar seguimento à obtenção de informações acerca da concepção dos moradores da comunidade sobre a enfermidade, bem como realizar os encaminhamentos necessários. O objetivo é desenvolver programas de intervenção e prevenção comunitária de doenças, com a convicção de que a ação da própria comunidade é a melhor forma de fomentar a saúde, entendida esta num sentido amplo, como um processo social (Pussieldi, Bruel, Brito, Hernandez \& Scarparo, 1996).

Como hipóteses básicas, temos que: 1) as características sócioeconômicas e culturais, tais como nível sócio-econômico, características da familia na infância do sujeito, número de filhos e situação sócio-laboral, estão significativamente relacionadas com os níveis de pressão arterial em comunidades carentes; e 2) que a relação entre as características sócio-culturais e a pressão arterial é mais relevante em comunidades com maiores estressores sociais.

\section{Método}

\section{Participantes}

Participaram da pesquisa, voluntariamente, 62 moradores da Vila Nossa Senhora de Fátima (Porto Alegre) (21 homems e 41 mulheres), com idades entre 14 e 75 anos $(m=41,3)$, de classe social entre média baixa e baixa. O grupo controle foi constituído por 289 participantes voluntários (120 homems e 169 mulheres), com idades entre 12 e 76 anos $(m=32)$, de classe social entre média e alta; em geral, professores, funcionários e estudantes da PUCRS.

\section{Procedimentos}

Para o grupo da Vila Fátima, os participantes foram selecionados através do processo de recadastramento para a setorização do atendimento nos postos de saúde do município (municipalização da saúde). Em grupos de dois, os entrevistadores batiam nas casas e perguntavam se as pessoas já haviam sido recadastradas. Se não, solicitava-se que a pessoa respondesse a um formulário com dados 
sobre a família. Em seguida, perguntava-se se a pessoa queria participar da pesquisa sobre pressão alta. Apenas uma pessoa negouse a participar.

No caso da amostra controle, os participantes dirigiam-se, voluntariamente, a um posto provisório instalado no Campus Central da PUCRS que, em um dia, levantou dados sobre índices cardíacos, durante a Semana da Solidariedade, sob a direção do Dr.Paolo Machado, da Unidade de Tratamento Intensivo (UTI) Coronária do Hospital São Lucas da PUCRS.

\section{Desenho e instrumentos}

O método utilizado parte de um desenho multidimensional com técnicas tanto quantitativas (correlacionais e de análise de variância) como qualitativas (análise de conteúdo), as quais são integradas na análise dos dados. Método este que foi utilizado eficazmente por um dos autores em estudo anterior (Gandarillas, 1995).

A fim de se levantar informações sobre variáveis constitucionais, sociais e fisiológicas, se construiu um questionário específico para esta pesquisa. As perguntas do questionário foram selecionadas de acordo com três fontes de informação: 1) Nos resultados da pesquisa sobre conceito de saúde, desenvolvida na Vila Nossa Sra. de Fátima (ver Pussieldi \& cols., 1996); 2) Na literatura específica sobre o tema em questão; e 3) Em contatos e conversas informais com hipertensos da Vila Fátima, especialmente no I Curso sobre Prevenção de Pressão Alta. O instrumento passou por pequenos estudos pilotos até sua versão definitiva. O questionário final inclui três grupos de variáveis (em ordem de apresentação): 1) Constitucionais e biológicas (sexo, idade, peso, tabagismo, conduta alcoólica, pré-existência de doenças, medicação); 2) Sócio-econômicas atuais (estado civil, número de filhos, anos morando na Vila Fátima, procedência, tipo de trabalho, formas de lazer, número de pessoas morando na casa, renda familiar, apoio e nível de perigo na comunidade, nível de educação); e 3) sócio-familares na infância (nível de afeto, disciplina e proteção dos cuidadores, número de irmãos, nível econômico da família durante a infância).

O questionário incluía perguntas a serem respondidas através de escalas de intervalo e nominais, além de várias questões abertas sobre as experiências da pessoa na sua infância, seus problemas atuais, seus planos para o futuro, relação com a comunidade e sobre suas crenças quanto à origem de seu estado de saúde. $\mathrm{O}$ entrevistador também fazia várias anotações acerca das próprias teorias dos participantes em relação a seu estado de saúde, observações relacionadas às condições da casa, à situação familiar e impressões sobre a personalidade do participante, bem como as possíveis causas de seu estado atual de saúde. Para medir a tensão utilizou-se um monitor digital de pressão arterial de método oscilométrico e inflado manual.

As perguntas do questionário para o grupo controle, devido ao fato de pertencer a um estudo com outros objetivos, não incluíam variáveis sócio-econômicas, apenas consitucionais e biológicas (idade, sexo, Índice Médio Corporal e fatores de risco, como tabagismo, etilismo, e obesidade).

\section{Procedimentos de coleta de dados}

A entrevista era realizada na própria casa dos participantes. Uma vez que a pessoa aceitava participar, o entrevistador realizava as perguntas do questionário, iniciando pela leitura e explicação do Consentimento Livre e Esclarecido, que incluia as informações pertinentes à confidencialidade da pesquisa e a encaminhamentos posteriores para o posto de saúde do Campus Aproximado, quando necessário. Uma vez dado o consentimento de participação eram abarcados, ainda, aspectos de prevenção e realizado o convite para que participassem dos grupos de Pressão alta realizados no posto. Ao término da entrevista, procedia-se a tomada da pressão sistólica, diastólica e a freqüência cardíaca com a pessoa sentada, pedindo-lhe o máximo relaxamento. A sessão demorava em torno de uma hora. O entrevistador voltava outro dia para realizar uma segunda medição onde, algumas vezes, se obtinha informação complementar que parecesse relevante para o estudo. Em seis casos não foi possível fazer uma re-medição em função da impossibilidade de encontrar a pessoa depois de várias visitas. $\mathrm{O}$ entrevistador era constante (um dos co-autores deste artigo), garantindo, assim, os mesmos critérios de levantamento de informação.

Cumprindo com as exigências da pesquisa com seres humanos, os objetivos da pesquisa foram explicados aos participantes, os quais davam autorização expressa quanto ao uso dos dados para fins científicos. Como esta pesquisa estava diretamente relacionada ao posto de saúde, bem como aos recursos oferecidos pelo Hospital São Lucas, as pessoas contavam com a garantia de encaminhamentos clínicos em casos mais graves. Em termos de garantias éticas, o projeto foi estudado e aprovado pelo orgão correspondente que, no caso desta pesquisa, era a Pós-graduação em Psicologia-PUCRS.

\section{Processamento de dados}

Os dados foram processados de três formas, de acordo com seu tipo: 1) As perguntas puramente quantitativas foram codificadas numericamente; 2) as variáveis qualitativas e perguntas abertas foram classificadas em categorias relevantes; e 3) alguns comentários relevantes foram respeitados em sua integridade original e incluidos como tal na discussão dos resultados. Assim, três níveis de análise foram utilizados: 1) Puramente quantitativo, com estatística multivariada; 2) Análise comparativa (pessoas com pressão alta X normal ou baixa) dos comentários das pessoas em relação a sua saúde; e 3) Integração quantitativa-qualitativa dos resultados. Em todas as análises, as médias de cada uma das medições da pressão foram tratadas como variáveis dependentes, e o restante das variáveis como variáveis independentes.

As seguintes análises foram realizadas:

1) Freqüência de hipertensos nas duas amostras (casos com pressão sistólica média acima de 160 e/ou diastólica acima de 95 $\mathrm{mmHg}$.

2) ANOVA comparando as duas amostras quanto à pressão arterial. Para isto, selecionou-se uma amostra aleatória de 65 pessoas 
retiradas da amostra do Campus Central da PUCRS. Realizou-se uma ANOVA sem a variável idade e outra neutralizando seu efeito (sendo tratada como co-variável).

3) Três regressões múltiplas na amostra da Vila Fátima, uma com variáveis constitucionais e biológicas, outra com variáveis sócioeconômicas atuais e outra com variáveis sócio-familiares da infância como preditores, e a pressão arterial média sistólica e diastólica como variáveis dependentes.

4) ANOVAS das variáveis significativas das regressões, com dois grupos feitos sobre cada variável baseados no percentil 50 de cada variável.

5) ANOVAS das seguintes variáveis, no caso da amostra da Vila Fátima: sexo, peso, tabagismo, conduta alcoólica, estado civil, procedência, tipo de trabalho e formas de lazer.

6) ANOVAS das seguintes variáveis, no caso da amostra do Campus Central da PUCRS: idade, peso, sexo, tabagismo, conduta alcoólica, sedentarismo e história familiar de doença cardíaca. No caso do peso, dois grupos foram formados com o ponto de corte 27,5 de Índice Médio Corporal, como critério de obesidade, similares a outros estudos em hipertensão (Duncan \& cols., 1993; Piccini \& Victora, 1994).

Os dados foram inseridos em micro-computador com o uso do programa EPIinfo, e as análises foram processadas com o pacote estatístico SPSSpc.

\section{Resultados}

A Tabela 1 mostra as características sócio-demográficas da amostra da Vila Fátima. Em relação à pressão arterial, o número de hipertensos na amostra da Vila Fátima foi de 9 sobre 62, o que significa 14,5\%. Sobre a amostra do Campus Central da PUCRS, 38 sobre 277, 13,7\%. As ANOVAS comparando as pressões sistólicas e diastólicas dos dois grupos não alcançaram níveis significativos $(\phi<0,05)$, nem na comparação simples nem na comparação com o efeito da idade excluído. As médias de pressão foram de $127,36 \mathrm{mmHg}$ (sistólica) e $78,57 \mathrm{mmHg}$ (diastólica) para o grupo da Vila Fátima, e 122,27mmHg e $80,24 \mathrm{mmHg}$ para o grupo do Campus Central.

A Tabela 2 mostra as regressões múltiplas das variáveis constitucionais e biológicas, sociais atuais, e sociais na infância, respectivamente, que alcançaram nível de significação, no grupo da Vila Fátima. Estas foram as seguintes: regressão com as variáveis constitucionais e biológicas predizendo a pressão sistólica média (PSM), com $R=0,32(F(1,60)=6,81, p<0,05)$ e Idade como preditor significativo $(t(59)=2,61, p<0,05)$; as mesmas variáveis predizendo a pressão diastólica média (PDM), com uma $R=0,26(F(1,60)=4,36$, $p<0,05)$ e Idade como preditor significativo ( $(59)=2,9, p<0,05)$; a regressão das variáveis sociais atuais predizendo PSM, com $R=0,30$ $(F(1,60)=6,81, p<0,05)$, e Número de Filhos como preditor significativo $(t(59)=2,61, p<0,05)$; as mesmas variáveis predizendo

Tabela 1

Características Sócio-demográficas do Grupo da Vila Nossa Senhora de Fátima

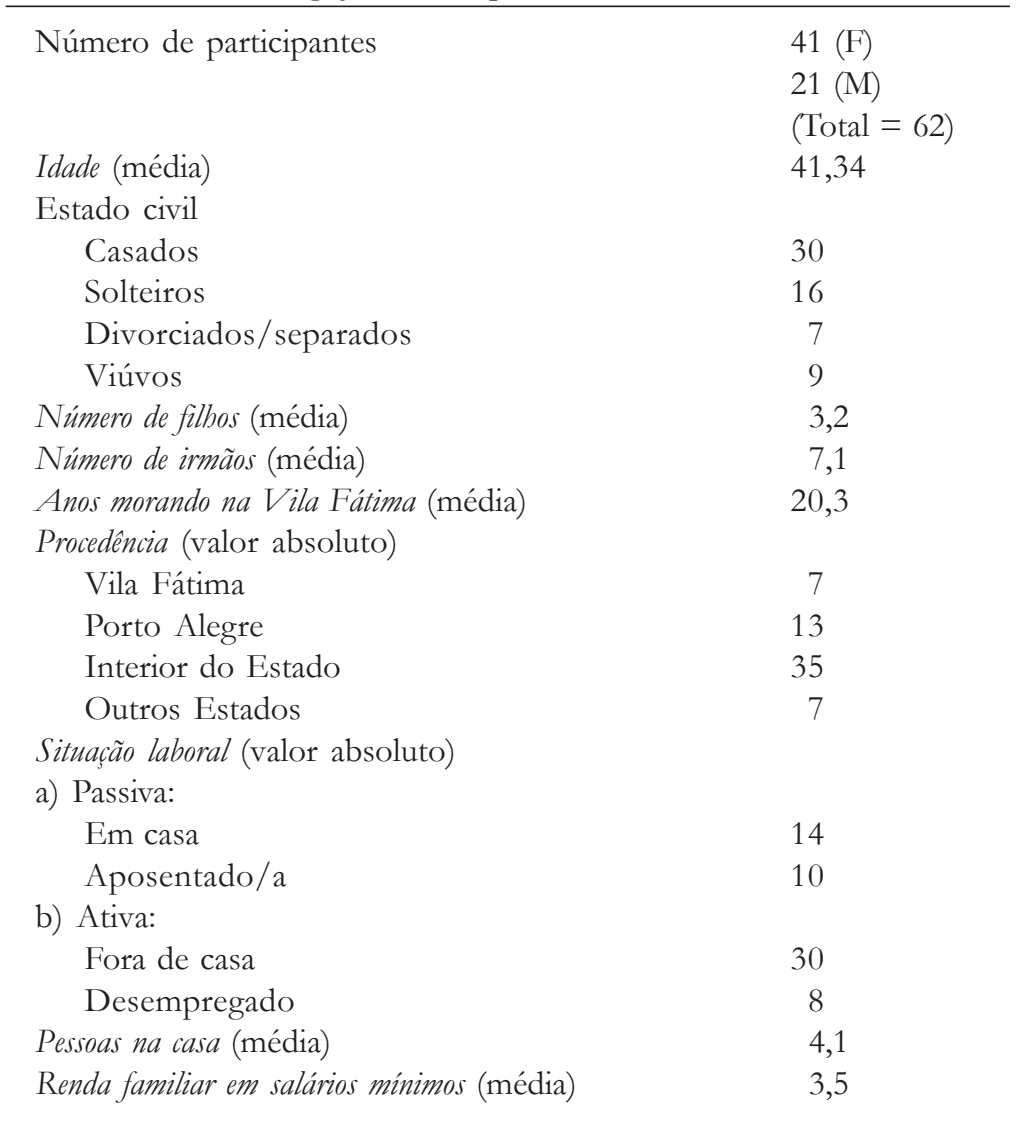


Tabela 2

Regressão Múltipla. Variáveis Constitucionais, Biológicas, Sociais Atuais: PSM e PDM. Variáveis

Sociais na Infância: PDM

\begin{tabular}{|c|c|c|}
\hline Preditores & PSM & PDM \\
\hline \multicolumn{3}{|l|}{ 1.Variáveis constitucuionais e biológicas $(n=62)$} \\
\hline Idade & $0,32 *$ & $0,26^{*}$ \\
\hline Peso & 0,20 & 0,15 \\
\hline Número de cigarros ao dia & 0,01 & 0,04 \\
\hline Freqüência de consumo de álcool & 0,10 & 0,00 \\
\hline$R$ & $0,32 *$ & $0,26^{*}$ \\
\hline \multicolumn{3}{|l|}{ 2.Variáveis sociais atuais $(n=61)$} \\
\hline Número de filhos & $0,30 *$ & $0,36 * *$ \\
\hline Anos na Vila Fátima & $-0,01$ & $-0,05$ \\
\hline Horas de trabalho remunerado & $-0,03$ & $-0,08$ \\
\hline Número de pessoas na casa & $-0,01$ & $-0,02$ \\
\hline Renda familiar & 0,09 & 0,06 \\
\hline Apoio da comunidade & 0,02 & $-0,06$ \\
\hline Perigo na comunidade & 0,20 & 0,14 \\
\hline$R$ & $0,30 *$ & $0,36^{* *}$ \\
\hline \multicolumn{3}{|l|}{ 3.Variáveis sociais na infância } \\
\hline Nível econômico familiar & $0,28 *$ & \\
\hline Número de irmãos & $-0,02$ & \\
\hline Apoio dos cuidadores & 0,09 & \\
\hline Castigo físico & $-0,21$ & \\
\hline Proteção dos cuidadores & 0,06 & \\
\hline
\end{tabular}

Nota.* $p<0,05 ; * * p<0,01$

Tabela 3

Diferenças Significativas em PSM e PDM- Niveis de Variáveis Constitucionais e Sócio-econômicas: Grupo da Vila Fátima

Idade sobre PSM

Idade sobre PDM

$\mathrm{N}^{\circ}$ de filhos sobre PDM

Nível econômico familiar na infância sobre PSM

Nível econômico familiar na infância sobre PDM

Trabalho

Nota. ${ }^{*} p<0,05 ; * * p<0,01$

PDM, com $R=0,36(F(1,60)=9,00, p<0,01)$, e Número de Filhos como preditor significativo $(t(59)=3,00, p<0,01)$; a regressão com as variáveis sociais da infância predizendo PDM, com $R=0,28$ $(F(1,58)=4,95, p<0,05)$, e Economia Familiar como preditor significativo $(t(57)=2,22, p<0,05)$.

A Tabela 3 mostra as ANOVAS significativas $(p<0,05)$ no grupo da Vila Fátima, que foram as seguintes para PSM como VD: Idade $(F(1,60)=10,26, p<0,01)$, nível econômico familiar na infância $(F(1,60)=4,68, p<0,05)$, e Trabalho $(F(1,60)=6,29, p<0,05)$. Os

\section{Níveis}

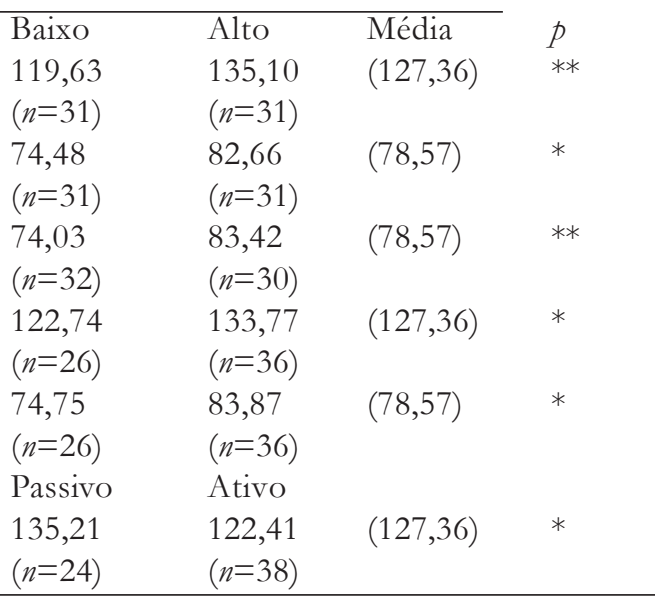

seguintes foram para a PDM: Idade $(F(1,60)=5,71, p<0,05)$, número de filhos $(F(1,60)=7,75, p<0,01)$, e nível econômico familiar na infância $(F(1,60)=7,06, p<0,01)$.

A Tabela 4 mostra as ANOVAS significativas para o grupo do Campus Central, que foram, como segue, para a PSM como VD: Sexo $(F(1,256)=7,49, p<0,01)$, Idade $(F(1,270)=19,96, p<0,001)$, Peso $(F(1,255)=24,06, p<0,001)$. No caso da PDM, foram: Sexo $(F(1,269)=7,36, p<0,01)$, Idade $(F(1,270)=21,54, p<0,001)$, e Peso $(F(1,255)=26,96, p<0,001)$. 
Tabela 4

\begin{tabular}{|c|c|c|c|c|}
\hline & \multicolumn{3}{|c|}{ Níveis } & \multirow[b]{2}{*}{$\mathrm{p}$} \\
\hline & Baixo & Alto & Média & \\
\hline Idade sobre PSM & $\begin{array}{l}117,31 \\
(n=131)\end{array}$ & $\begin{array}{l}127,22 \\
(n=141)\end{array}$ & $\begin{array}{l}122,31 \\
(n=272)\end{array}$ & $* * *$ \\
\hline Idade sobre PDM & $\begin{array}{l}76,72 \\
(n=131)\end{array}$ & $\begin{array}{l}83,67 \\
(n=141)\end{array}$ & $\begin{array}{l}80,32 \\
(n=272)\end{array}$ & $* * *$ \\
\hline Peso sobre PSM & $\begin{array}{l}118,20 \\
(n=184)\end{array}$ & $\begin{array}{l}129,32 \\
(n=73)\end{array}$ & $\begin{array}{l}121,35 \\
(n=257)\end{array}$ & $* * *$ \\
\hline Peso sobre PDM & $\begin{array}{l}77,76 \\
(n=184) \\
\text { Feminino }\end{array}$ & $\begin{array}{l}86,16 \\
(n=73) \\
\text { Masculino }\end{array}$ & $\begin{array}{l}80,15 \\
(n=257)\end{array}$ & $* * *$ \\
\hline Sexo sobre PSM & $\begin{array}{l}119,67 \\
(n=157)\end{array}$ & $\begin{array}{l}126,14 \\
(n=114)\end{array}$ & $\begin{array}{l}122,39 \\
(n=271)\end{array}$ & $* *$ \\
\hline Sexo sobre PDM & $\begin{array}{l}78,59 \\
(n=157)\end{array}$ & $\begin{array}{l}82,81 \\
(n=114)\end{array}$ & $\begin{array}{l}80,36 \\
(n=271)\end{array}$ & ** \\
\hline
\end{tabular}

Nota. $* p<0,05 ; * * p<0,01 ; * * * p<0,001$

\section{Discussão}

Os resultados sobre a incidência de hipertensão na Vila Fátima (14,5\%) fornecem índices similares aos do grupo controle do Campus Central da PUCRS (13,7\%), e semelhantes aos do estudo de Duncan e colaboradores (1993) em Porto Alegre (15\%), embora um tanto diferentes dos 19,8\% encontrados por Piccini e Victora (1994) em Pelotas (RS). Mais ainda, este último estudo assinalou uma diferença significativa da incidência da hipertensão entre classes sociais, mostrando as classes mais desfavorecidas economicamente com mais altos índices de hipertensão (entre 23 e 25\%). Dada a repetida constatação, em outros estudos (Costa, 1983; Krantz, 2002), sobre as diferenças na incidência de hipertensão entre classes sociais, os resultados devem ser tomados com cautela. A existência de variáveis intervenientes, como a diferença do ambiente no qual se media a pressão (na Vila Fátima na própria casa dos participantes, e no Campus Central, na sala de recepção de um departemanto), e o tempo de relaxamento antes da medição da pressão (em torno de meia hora para o grupo da Vila Fátima e alguns minutos no caso do grupo do Campus Central) no levantamento dos dados sobre pressão arterial, poderiam ter afetado os resultados. Uma explicação alternativa pode ser a de que fatores sociais como o nível sócio-econômico, não afeta de forma direta a incidência da hipertensão, senão que demonstra a importância relativa do ambiente no desenvolvimento da hipertensão. Dessa forma, variáveis como idade, sexo e peso aparecem mais relevantemente associadas ao aparecimento de hipertensão na amostra do Campus Central que na amostra da Vila Fátima. Os resultados sugerem, assim, que em comunidades mais carentes, as características sócio-econômicas podem explicar melhor o desenvolvimento da hipertensão do que em comunidades mais favorecidas. Este dado corrobora a afimação de Harrap (1994) de que a contribuição genética $v$ s. ambiental à hipertensão varia através dos grupos sociais.
As regressões mostram a grande importância da idade como variável constitucional, fundamental na explicação da hipertensão. De qualquer forma, e como assinalamos anteriormente, a idade, por si mesma, talvez não seja a única explicação desta relevância. Talvez estressores sociais e psicológicos relacionados com a idade nesta comunidade e em outros grupos sociais possam, também, dar conta, em parte, deste nível de significância da variável idade. Assim, poderíamos explicar porque uma grande proporção das pessoas mais velhas não desenvolva hipertensão. Em relação às variáveis sociais da vida atual da pessoa, é um tanto surpreendente a força da variável número de filhos na relação com a pressão arterial, especialmente na pressão diastólica. O estress relacionado com a diminuição do controle dos pais sobre a família e a casa em famílias numerosas poderia dar conta desta relevância. A relação entre a importância dos baixos níveis de controle social e a hipertensão tem sido reportada na literatura (Bosma \& cols., 1997; De Oliveira, 2000, especialmente no que se refere a estresse mental; Karasek \& Theorell, 1990, no contexto laboral). Finalmente, esclarecedores são os resultados sobre a relação entre economia familiar do tempo de infância e a pressão arterial. Quanto melhor economicamente a pessoa percebe a situação familiar na infância, mais altos são os índices de pressão diastólica. Isso pode indicar que não é tanto a pobreza quanto a percepção relativa desta que pode relacionar-se com o desenvolvimento de pressão alta, sublinhando a importância dos fatores emocionais. Estes resultados podem ser interpretáveis a partir do conceito do consonância cultural (Dressler \& Santos, 2000). Pessoas que vivenciaram uma situação sócio-econômica melhor em sua infância podem perceber sua vida atual como mais afastada da consonância cultural que as pessoas que têm vivido em um mesmo status sócio-econômico durante todo o decorrer de suas vidas. Os resultados, à luz desta interpretação, apoiariam a relação entre consonância cultural e níveis de pressão arterial, reportada por Dressler e colaboradores (2001).

As ANOVAS sublinham a importância destas três variáveis na incidência de hipertensão. É de se destacar aqui como as 
variáveis sociais são melhor refletidas na pressão diastólica do que na sistólica, apoiando outros estudos que indicam a pressão diastólica como mais influenciada por fatores sócio-emocionais (Kalimo \& Vuori, 1993; Knox, 1993). Uma exceção, neste estudo, é quanto ao tipo de atividade laboral. A maior pressão sistólica aparece nas pessoas de população passiva comparada com as de populaçao ativa. Isso não só refere-se aos aposentados, mas também às donas de casa. A sensação expressada por estas últimas, de estarem fechadas em casa, de não poderem controlar e transformar certos aspectos de sua realidade, poderia estar relacionada com o desenvolvimento da hipertensão.

O indivíduo passa, portanto, através desta passividade, a se perceber alienado do processo social, um estrangeiro em seu próprio ambiente. Podemos pensar que este é um fenômeno comum quando falamos em classes populares, porém está associado a uma interpretação histórica linear do cotidiano. Segundo Jelin (1994), esta forma de interpretação é fundamentalmente fatalista. Está presente a idéia de desigualdade social, entendida, muitas vezes, até como injustiça, porém esta consciência desaparece na medida em que a ideologia dominante prega a idéia de desgraça inevitável e sorte como determinantes das crises sociais.

Até aqui, através dos resultados quantitativos, temos uma descrição da pessoa de pressão mais alta como percebendo que sua situação econômica tem piorado com a idade, talvez configurando um processo de marginalização. Além disso, identificamos uma pessoa que passa mais tempo em casa, dedicando-se aos trabalhos domésticos, possivelmente responsável por um elevado numero de filhos(as), o que poderia, igualmente, contribuir para uma sensação de falta de controle sobre seu destino e conseqüente impotência em mudar para melhor. Os resultados qualitativos sobre perguntas abertas e estudo de casos nos proporcionam uma visão mais profunda acerca desses sentimentos das pessoas hipertensas.

Em geral, uma das manifestações mais recorrentes dos hipertensos, em comparação com os não hipertensos, é a ausência de problemas, o que eles expressam superficialmente, e a falta de planos ou expectativas para o futuro. Foram comuns, entre os hipertensos entrevistados, os comentários do tipo estou feliz. com vida, e só espero que tudo continue como sempre foi. Mas quando as perguntas vão se focalizando, aparece um quadro completamente contrário, de uma pessoa dominada por problemas e preocupações constantes. Este é o caso de uma mulher de 60 anos com uma PSM (pressão sistólica média) de 199 e PDM (pressão diastólica média) de $88 \mathrm{mmHg}$. Apesar da grande lista de medicação anti-hipertensão que parece consumir, ela manifesta não ter problemas e não ter planos para o futuro, apenas espera que fique tudo como está. Porém, ao perguntarmos porque ela acha que tem pressão alta, responde que não se pode evitar as preocupações. Outro caso característico é o de um homem de 61 anos, recuperando-se de um derrame na perna, devido, em parte, à pressão alta (PSM de 167 e PDM de 96), que refere que a vida sempre the foi boa e a única coisa que espera é que tudo siga assim. Mas ao ter sua pressão medida e constatar que está alta, apesar da medicação anti-hipertensão, começa a chorar e a relacionar o problema da pressão alta com suas preocupações em relação à sua vida, à família e, especialmente, a um filho com quem tem conflitos, porque, de acordo com ele, só pensa nele quando precisa de dinheiro para drogas.

O hipertenso, neste estudo parece querer se afastar, evitar ou ocultar o que talvez considere como intrínseco à vida, uma preocupação vital. É o caso de uma senhora de 59 anos, mãe de 9 filhos, tomando várias medicações anti-hipertensão, na época da entrevista com PSM 142 e com uma situação econômica bem melhor na infância, que explica, resignadamente: a gente mora onde Deus coloca. Sua filha, não hipertensa e ativa comunitariamente refere: para a mãe tudo está bom. O hipertenso, apesar da vida, está feliz: Por isso quando se aprofunda mais em suas concepções de vida, aparecem todos os problemas. A vida aparece, intrinsecamente, como uma contínua ameaça. Uma mãe de 45 anos (PSM 158, PDM 121) coloca que tudo são problemas, e que se encontra muito preocupada com as coisas da vida. Outra senhora, de 66 anos (PSM 176, PDM 95), mora sozinha com o marido, que passa das 6 da manhã até às 10 da noite fora de casa, trabalhando. A preocupação maior desta senhora é que algo aconteça ao marido quando sai para trabalhar. Também fica doente dos nervos pelos episódios de violência e perigo na Vila o que relaciona com a pressão alta.

A maioria dos hipertensos faz uma associação entre os nervos e a hipertensão. Um homem de 51 anos (PSM 158, PDM 105) refere que seus problemas são de nervos devido ao trabalho em excesso no passado. Para a aposentada de 64 anos, com 8 filhos, a pressão alta deve-se ao fato de ficar irritada com o comportamento dos filhos e netos.

Segundo Souza (citado em Costa, 1989), a doença dos nervos (assim denominada em função de representações que permitem ao indivíduo atribuir sentido e naturalizar essa sensação generalizada de impotência e mal-estar que se faz presente) é uma forma de sobrevivência, pois permite ao indivíduo, principalmente, de baixa renda um entendimento causal lógico daquelas estruturas superiores a ele que, justamente por entremearem sua existência, tornam-se intoleráveis e, portanto, afastadas de qualquer possibilidade de decodificação. Entretanto, seu corpo começa a sinalizar e, neste sentido, a denominação de doença dos nervos não é mera casualidade se compreendermos, como Costa (1989), que "não existe doença independente de seu modo de expressão. A idéia de causalidade faz parte da maneira como a doença se constrói. A representação da causalidade é indissociável da subjetividade que a produz" (p. 20).

A solidão e carência afetiva também parecem estar por trás de muitos destes casos. A mãe de 7 filhos, 48 anos, com PSM de 173 e PDM de 97 (apesar da medicação anti-hipertensão) manifesta ter tido uma relação fria com os pais na infância. Isto parece ter continuado pela vida afora. Está há 24 anos morando sem companheiro, separada de seu marido. Ela mostra orgulho da sua grande coleção de bonecos, todos bem ordenados na entrada da casa, como se estivessem dando uma calorosa 
recepção aos visitantes. Ela acha que seu problema com a pressão alta é por comer demais (ela demonstra obesidade). Refere que tenta controlar-se, mas não pode, porque, muitas vezes, fica ansiosa em casa e come. À pergunta de por que ela fica ansiosa, não sabe bem o quê responder. Mas a carência afetiva e o vazio na vida por trás dessa ansiedade é uma hipótese inevitável. Um caso similar é o de uma senhora de 62 anos, com uma hipertensão leve (PSM 148), que vive com a filha deficiente mental, sendo sua única cuidadora e que anda preocupada porque pensa que um vizinho quer invadir seu terreno. Mas a falta quase total de contato com os vizinhos poderia estar subjacente a esta postura defensiva. Esta falta de contato social forçada ou por isolamento voluntário dos hipertensos que participaram deste estudo relaciona-se à incontestável importância do apoio social como fator protetor da hipertensão arterial, conforme encontramos na literatura (Knox, 1993; Krantz, 2002).

Assim, a fotografia das características sociais e emocionais do hipertenso típico, em nosso estudo, é a do indivíduo que chegou à Vila Fátima, decrescendo em sua situação sócio-econômica, e entrou numa situação economicamente passiva, com menor apoio social, poucas oportunidades, percebidas ou reais, de transformar a sua vida e o seu ambiente (aproximando-se a sua consonância cultural, cf. Dressler $\&$ Santos, 2000). Essa percepção de falta de controle e poder sobre sua vida une-se a uma ampla dedicação para com os filhos. Quanto às preocupações inerentes à sua vida, observamos que estas são abordadas através da resignação, a evitação, a fuga dos problemas, a negação da realidade, o afastamento do seu contexto e, decorrente disso, uma transposição perversa para um afastamento de seu próprio corpo. Produz-se, assim, um círculo vicioso: quanto mais preocupações ou estresse, mais afastamento, e quanto mais afastamento do entorno social (menor apoio social) e de si mesmo, menor controle percebido e mais preocupante é a percepção da realidade, o que acarreta maior afastamento. Tudo isso contribui para que a pessoa mantenha um controle defensivo e inadequado sobre um corpo que é sentido, continuamente, como a ponto de ser perdido. Associado a isso, ocorre uma incapacidade de ativação adaptativa do sistema parassimpático e uma excessiva e descontrolada ativação crônica do SNS (Cacioppo, 1994; Gray 1971; Kemper, 1986), o que pode explicar o desenvolvimento da hipertensão.

Evidencia-se, assim, como as circunstâncias sociais e econômicas podem levar a uma atitude de passividade frente à vida e de alienação em relação ao entorno social, a si próprio e ao seu corpo. Entretanto, talvez não seja apenas a situação econômica e os aspectos emocionais, mas também a ideologia e cultura dominantes em nossa sociedade. A cultura competitiva imperante prima pela responsabilidade individual do fracasso, que leva consigo a estigmatização e o isolamento do indivíduo mal sucedido, o qual interiorizará este fracasso e se auto-alienará, afastando-se, ainda mais, de uma consonância cultural. Esse sujeito também aprenderá que a culpa pelo fracasso é a falta de controle sobre si mesmo. Da percepção de fracasso e de impotência desenvolve uma postura defensiva e auto-destrutiva e, conseqüentemente, uma perda de poder sobre seu corpo que é, em parte, entregue ao poder do sistema social.

Neste sentido, Arendt (1965, citado em Jelin, 1994) expressa que

podemos considerar o desaparecimento do gosto pela liberdade política como a retirada do indivíduo a uma esfera íntima de consciência, onde encontra a única região apropriada para a liberdade humana; essa região, como fortaleza ruída, será a base a partir da qual o indivíduo, tendo prevalecido sobre o cidadão, se defenderá, então, contra uma sociedade que, por sua vez, prevalece sobre a individualidade. (p. 45)

Assim, observamos como os moradores da Vila Fátima percebem que a sua saúde, entendida como ausência de doença ou de dor, é, em parte, responsabilidade dos profissionais da saúde (Pussieldi \& cols., 1996). Isto é ilustrado por um membro do grupo de hipertensos que reclama que eles tem que fazer o

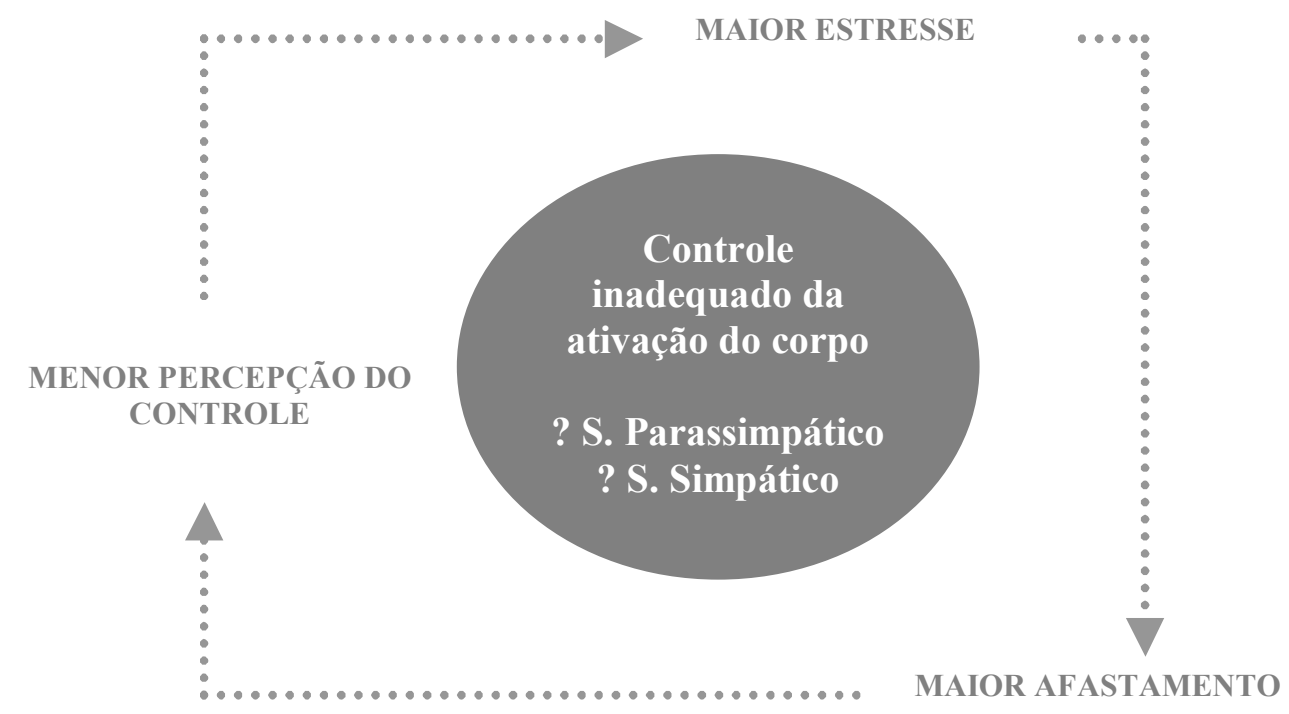

Figura 1. Dinâmica social da hipertensão crônica. 
que os médicos mandam, porque eles tem a autoridade. $\mathrm{O}$ próprio ambiente ecológico é percebido como responsabilidade das autoridades, portanto, cabe somente a elas melhorar essa situação caótica (Pussieldi \& cols., 1996).

Analisados em sua globalidade, os resultados aqui apresentados apóiam, em geral, as hipóteses principais deste estudo, embasadas nos modelos teóricos de Gray (1971), Cacioppo (1994) e Kemper (1986). Variáveis sociais indicativas de carências no momento presente aparecem, neste estudo, tanto quantitativa como qualitativamente, relacionadas com a hipertensão em geral. Dessa forma, hipertensão poderia estar associada a fatores sociais que predispõem a uma diminuição crônica da atividade do sistema nervoso parassimpático (Ex.: a diminuição de fontes de apoio social e status social) e a um aumento da ativação crônica do sistema nervoso simpático (Ex.: a falta de controle sobre o entorno social e o próprio corpo). Por outro lado, variáveis de criação na infância não aparecem, neste caso, com a mesma relevância outorgada por outros estudos sobre a saúde fisiológica (Fowles, 1980; Kalimo \& Vuori, 1993; Knox, 1993; Larsen \& Ketelaar, 1991; Polanky \& cols., 1985; Raine, 1988; Theorell \& Emlund, 1993; Whiting \& Whiting, 1975). Seria importante, em futuras pesquisas, aprofundar as razões acerca do por quê que em situações similares, algumas pessoas desenvolvem hipertensão e outras não. Nessa linha, este estudo sugere que pode existir uma relação no desenvolvimento de fatores psicológicos, como locus de controle externo, indefensão aprendida e baixa auto-eficácia percebida sobre a própria saúde com a hipertensão arterial crônica.

É importante assinalar que o delineamento e a amostra escolhidos neste estudo permitem destacar variáveis sociais que podem afetar a hipertensão. Em nenhum momento, neste artigo, pretendeu-se entender os fatores sociais como necessários ou suficientes para o desenvolvimento da hipertensão. Dessa forma, outros estudos com diferentes amostras, seriam necessários para que pudéssemos obter maior compreensão acerca da importância relativa dos fatores sociais na hipertensão em se tratando da população em geral.

\section{Conclusões e Recomendações de Ação}

Esta pesquisa apresenta um enfoque global da saúde, entendida não no sentido meramente médico, mas na relação do indivíduo com seu meio social e ecológico. A saúde é conceitualizada como um processo social que se origina da integração entre indivíduo e meio. Através deste enfoque, tanto os métodos quantitativos como qualitativos são usados e integrados em uma visão global, possibilitando uma compreensão mais completa e integrada do indivíduo inserido em seu meio social e ecológico. Assim, observamos que este meio parece contribuir mais para o desenvolvimento da hipertensão em comunidades com maiores estressores sócioeconômicos. Características sociais e familiares como o número de filhos, a degradação sócio-econômica e a situação laboral, combinam-se com características constitucionais como a idade, e características emocionais, como a atitude passiva ante o entorno e a projeção dos problemas sociais para si mesmo e seu corpo, além das mensagens ideológicas e culturais de afastamento de sua propria natureza, presentes em nosso contexto.

No momento de o profissional da saúde intervir para prevenir a incidência da hipertensão, várias são as recomendações que surgem do trabalho desta pesquisa:

1) Fomentar a concepção do corpo como ativo e mostrar as possibilidades de ter uma relação construtiva, positiva, livre, e espontânea com ele. Sempre evitar ter uma atitude paternalista e hierárquica geradora de dependência, e sim de cooperação com a pessoa.

2) Fomentar a participação da pessoa em associações ou grupos comunitários. Animar a pessoa a sair periodicamente de seu ambiente mais imediato para obter uma visão mais fidedigna de seu meio.

3) Fomentar a interrelação positiva e construtiva da pessoa com seu ambiente ecológico, entendendo, aqui, ecologia como o ecossistema social e ambiental do entorno da pessoa (Bronfenbrenner, 1996). Mostrar a importância de ter uma relação equilibrada, saudável, livre e espontânea com seu entorno. Fomentar a identificação da pessoa com os aspetos construtivos desta relação com seu meio.

Uma grande proporção dos moradores da Vila Fátima sentese despossuída, marginalizada. A volta a uma relação natural com sua ecologia urbana pode fortalecer a sensação de pertencer a um lugar ao mesmo tempo que favorece a relação construtiva do indivíduo com seu meio. A maioria dos moradores da Vila Fátima procede de áreas rurais do interior do estado. Quando chegaram à capital, num intento desesperado de adaptação ao meio urbano, deixaram para trás costumes e concepções ancestrais e tradicionais de autocuidado ou cuidado mútuo, sem que fossem compensados por outras estratégias de otimização de recursos. Embora alguns costumes não sejam válidos na vida moderna urbana, outros poderiam ser muito valiosos para a vida atual destes moradores. A recuperação de algumas concepções tradicionais quanto à relação natural e à identificação com o meio poderia indicar uma solução contra a alienação, o medo à propria natureza, e a repressão desta. Este pode ser o exemplo, em nosso estudo, da senhora de 72 anos, analfabeta, de Cachoeira do Sul (RS), que, de acordo com ela, trabalhou muito na infância com os pais na roça. Quando o médico lhe diagnosticou hipertensão, ela começou a cultivar, nos $2 \mathrm{~m}^{2}$ que tem na entrada de sua casa, plantas que, na tradição de sua região, eram boas em chá para a circulação de sangue. Agora, com PSM de 127 e PDM de 74, tem a pressão sob controle, e possui uma grande variedade de plantas medicinais trazidas de sua região nesse minúsculo pedaço de terra que mostra com orgulho, e, inclusive, distribui entre os vizinhos com doenças. Agora, o que mais gostaria seria ter mais espaço para aumentar sua pequena horta com novas plantas.

\section{Referências}

Anderson, N. B., McNeilly, M. \& Myers, H. (1991). Autonomic reactivity and hypertension in blacks: A review and proposed model. Ethnic Disorders, 1, 154-170.

Bosma, H., Marmot, M. G., Hemingway, H., Nicholson, A., Brunner, E. J. \& cols. (1997). Low job control and risk of coronary heart disease in the Whilehwall II (prospedctive cohort) study. American Journal of Public Health, 86, 332-340. 
Bronfenbrenner, U. (1979/1996). A ecologia do desenvolvimento humano. Porto Alegre: Artes Médicas.

Cacioppo, J. T. (1994). Social neuroscience: Autonomic, neuroendocrine, and immune responses to stress. Pychophysiology, 31, 113-128.

Consolim-Colombo, F. M. \& Krieger, E. M. (2000). Sistema nervoso simpático e hipertensão arterial. Hipertensão, 3, 86-89.

Costa E. A. (1983). Hipertensão Arterial como problema de massa no Brasil: Caracteres epidemiológicos e fatores de risco. Ciência e Cultura, 35, 1642-1649.

Costa, J. (1989). Psicanálise e contexto cultural. Rio de Janeiro: Campus.

De Oliveira, W. (2000). Estresse mental como fator de risco para a hipertensão arterial. Hipertensão, 3, 107-109.

Dressler, W. W., Balieiro, M. C. \& Santos, J. E. (2001). Culture, skin color, and arterial blood pressure in Brazil. American Journal of Human Biology, 11, 49-59.

Dressler, W. W. \& Santos, J. E. (2000). Dimensões sociais e culturais da hipertensão no Brasil: Uma revisão. Cadernos de Saúde Pública, 16, 303-315.

Dressler, W. W., Balieiro, M. C. \& Santos, J. E. (1998). Culture, socioeconomic status, and physical and mental health in Brazil. Medical Antbropology Quaterly, 12, 424-446.

Duncan, B. B., Schmidt, M. I., Polanczyk, C. A., Homrich, C. S., Rosa, R. S. \& Aachutti, A. C. (1993). Fatores de risco para doenças não-transmissíveis em área metropolitana na região sul do Brasil. Prevalência e simultaneidade. Revista de Saúde Publica, 27, 143-148.

Erickson, M. F., Egeland, B. \& Pianta, R. (1989). Effects of maltreatment on the development of young children. Em D. Cicchetti \& V. Carlson (Orgs.), Child maltreatment: Theory and research on the causes and consequences of child abuse and neglect (pp. 647-684). Cambridge: Cambridge University Press.

Fowles, D. C. (1980). The three arousal model: Implications of Gray's two-factor learning theory for heart rate, electrodermal activity, and psychopathy. Psychophysiology, 17, 87-104.

Gandarillas, M. A. (1995). Cultura y fisiologia: El papel de la crianza infantil en la actividad psico-fisiologica. Un estudio inter-disciplinar. Tese de Doutorado nãopublicada, Universidad Autonoma de Madrid. Madrid, Espanha.

Gray J.A. (1971). The psychophysiological basis of introversion-extraversion. Behavior Research and Theory, 8, 249-266.

Gray, J. A. (1981). A critique of Eysenck's theory of personality. Em H. J. Eysenck (Org.), A model for personality (pp. 246-276). New York: Springer.

Harrap, S. B. (1994). Hypertension: Genes versus environment. The Lance, 344, 169-171.

Jelin, E. (1994). Construir a cidadania: Uma visão desde baixo. Lua Nova - Cultura e Politica, 32, 39-57.
Kalimo, R. \& Vuori, J. (1993). Psychosocial factors in youth and at working age as predictors of blood pressure: A prospective study. Journal of Psychosomatic Research, 37, 643-652.

Karasek, R. A. \& Theorell, T. (1990). Healthy work, stress, productivity, and the reconstruction of working life. New York: Basic Books.

Kemper, T. D. (1986). How many emotionas are there? Wedding the social and the autonomic components. American Journal of Sociology, 93, 263-289.

Knox, S. S. (1993). Perception of social emotional warmth and blood pressure in young men. Perceptual and Motor Skills, 77, 132-134.

Krantz, D. S. (2002). Effects of psychological and social factors on organic disease: A critical assessment of research on coronary heart disease. Annual Review or Psychology, 53, 321-369.

Larsen, R. J. \& Ketelaar, T. (1991). Personality and susceptibility to positive and negative emotional states. Journal of Personality and Social Psychology, 61, 132140.

Linden, W., Chambers, L., Maurice, J. \& Lenz, J. (1993). Sex differences in social emotional warmth, self-deception, hostility, and ambulatory cardiovascular activity. Health Psychology, 12, 376-380.

Peisner, E. (1989). To spare or not to spare the rod. Em J. Valsiner (Org.), Child development in cultural context (pp. 111-141). Lewiston, NY: Hogrefe and Huber.

Piccini, R. X. \& Victora, C. G. (1994). Hipertensão arterial sistêmica em área urbana no sul do Brasil: Prevalência e fatores de risco. Revista de Saúde Pública, 28, 261267.

Polanky, N. A, Gaudin, J. M., Amons, P. W. \& Davis, K. B. (1985). The psychological ecology of the neglectful mother. Child abuse and Neglect, 9, 265-275.

Pussieldi, M. H., Bruel, T. C. S., Brito, R. C., Hernandez, A. R. C. \& Scarparo, H. (1996). Saúde comunitária: Uma perspectiva de grupos da Vila Nossa Senhora de Fátima. Psico, 27, 63-77.

Raine, A. (1988). Antisocial behavior and social psychophysiology. Em H. L. Wagner (Org.), Social psychophysiology and emotions: Theory and clinical applications (pp. 231-250). Chichester: J. Wiley \& Sons.

Theorell, T. \& Emlund, N. (1993). On physiological effects of positive and negative life changes: A longitudinal study. Journal of Psychosomatic Research, 37, 653659.

Whiting B. B. \& Whiting J. W. M. (1975). Children of six cultures: A psychocultural analysis. Cambridge, MA: Harvard University Press.

Recebido: 08/11/2001

Última revisão: 27/01/2004

Aceite final: 04/03/2004

Sobre os autores

Miguel Angel Gandarillas é Psicólogo. Mestre e Doutor em Psicologia pela Universidade Autônoma de Madri. É Diretor da Facultad de Psicología, Universidad S.E.K, Segovia, España.

Sheila Gonçalves Câmara é Mestre e Doutora em Psicologia Social e da Personalidade pela Pontifícia Universidade Católica do Rio Grande do Sul. É Docente da área de Psicologia Social e Comunitária do Curso de Psicologia da Universidade Luterana do Brasil.

Helena Scarparo é Psicóloga. Mestre em Educação e Doutora em Psicologia. Docente da Faculdade de Psicologia da Pontifícia Universidade Católica do Rio Grande do Sul. 